\title{
1 Novel RT-ddPCR Assays for determining the transcriptional profile of SARS-CoV-2
}

2

3 Sushama Telwatte ${ }^{a, b}$, Nitasha Kumar, ${ }^{a, b}$, Albert Vallejo-Gracia ${ }^{c}$, G. Renuka Kumar ${ }^{c}$, Chuanyi

$4 \quad$ M. Lu ${ }^{a, b}$, Melanie Ott ${ }^{a, c}$, Joseph K. Wong ${ }^{a, b}$, Steven A. Yuklª,b

5

6 a. Department of Medicine, University of California, San Francisco, CA, USA

7 b. Department of Infectious Diseases, San Francisco VA Health Care System, San

8 Francisco, CA, USA

9 c. Gladstone Institute of Virology, Gladstone Institutes, San Francisco, CA, USA

10 *Corresponding author: Steven Yukl; San Francisco VA Medical Center, 4150 Clement St,

11 111W3, San Francisco, CA 94121, USA; steven.yukl@ucsf.edu

12

13 Declarations of interest: none 


\section{Highlights}

- We developed a novel panel of 7 quantitative RT-ddPCRs assays for SARS-Cov-2

- Our panel targets nongenic and genic regions in genomic and subgenomic RNAs

- All assays detect 1-10 copies and are linear over 3-4 orders of magnitude

- All assays correlated with the clinical Abbott SARS-CoV-2 Viral Load Assay

- Clinical samples showed higher copy numbers for targets at the 3' end of the genome

\section{Abstract}

The exact mechanism of coronavirus replication and transcription is not fully understood; however, a hallmark of coronavirus transcription is the generation of negativesense RNA intermediates that serve as the templates for the synthesis of positive-sense genomic RNA (gRNA) and an array of subgenomic mRNAs (sgRNAs) encompassing sequences arising from discontinuous transcription.

Existing PCR-based diagnostic assays for SAR-CoV-2 are qualitative or semiquantitative and do not provide the resolution needed to assess the complex transcription dynamics of SARS-CoV-2 over the course of infection. We developed and validated a novel panel of specially designed SARS-CoV-2 ddPCR-based assays to map the viral transcription profile. Application of these assays to clinically relevant samples will enhance our understanding of SARS-CoV-2 replication and transcription and may also inform the development of improved diagnostic tools and therapeutics.

37 Key words: SARS-CoV-2; droplet-digital PCR; quantitative assays; coronavirus; viral 


\section{Introduction}

The etiologic agent responsible for the ongoing COVID-19 pandemic, identified as Severe Acute Respiratory Syndrome Coronavirus 2 (SARS-CoV-2), ${ }^{1,2}$ is an enveloped virus with a positive-sense, single-stranded RNA genome of $\sim 30 \mathrm{~kb}$ and is a member of the $\beta$ coronavirus genus. SARS-CoV-2, which is the seventh coronavirus known to infect humans, shares approximately $50 \%$ sequence homology with MERS and $79 \%$ sequence homology with SARS-CoV ${ }^{3}$ but appears to be more closely related to the SARS-like bat coronaviruses RmYN02 from R. malayanus and RaTG13 from R. affinis $(93.3 \%$ and $96.1 \%$ sequence identity, respectively), ${ }^{4}$ though its origin is, to date, unsettled ${ }^{5,6}$.

The exact mechanism of SARS-CoV-2 replication and transcription is not fully understood; however, a hallmark of coronavirus transcription and other viruses of the order Nidovirales is the generation of negative-sense RNA intermediates that serve as the templates for the synthesis of positive-sense genomic RNA (gRNA) and an array of subgenomic RNAs (sgRNAs), which arise from discontinuous transcription and encompass sequences from both ends of the genome ${ }^{7,8}$ (Fig. 1). Following cell entry, SARS-CoV-2 genomic RNA is transcribed and translated to generate the nonstructural proteins (NSP) from the two open reading frames (ORF), ORF1a and ORF1 $\mathrm{b}^{8}$, a process thought to involve the virus replication complex, transcription-regulating sequences (TRSs), the $\mathrm{N}$ protein, and double membrane vesicles in the cytoplasm of infected cells $s^{9-11}$. During the synthesis of the negative strand RNA, sgRNAs arise from a template switch that adds a copy of the 'leader' sequence ( 70 nucleotides in the 5' untranslated region [UTR] containing a short transcription-regulating sequence [TRS] at the 3 ' end) to the 'body' sequence derived from one of various genes in the 3 ' third of the genome (including genes for structural proteins) ${ }^{12-14}$. Transcription of the sgRNAs is likely regulated by TRS sequences in the leader sequence and upstream of 3 ' genes ${ }^{9}$, and may allow for greater expression of certain viral genes.

A recently published study confirms that a similar mechanism exists for SARS-CoV-2 to generate nine canonical sgRNAs distinct from genomic $\mathrm{RNA}^{8}$ (Fig. 1). For other coronaviruses, sgRNAs encode virulence factors such as proteins that directly cause lesions ${ }^{15}$ 
67 or indirectly inhibit immune responses ${ }^{16}$. Incorporation of $5^{\prime}$ UTR sequences into the capped 68 subgenomic mRNA templates of SARS-CoV may confer resistance to cleavage by viral nsp1

69 protein $^{17}$, which typically inhibits host gene expression by degradation of host mRNA ${ }^{18-20}$. For 70 positive-sense RNA viruses, sgRNAs act as messengers for expression of structural proteins 71 or proteins related to pathogenesis and can regulate the transition between translation and 72 virion production ${ }^{21}$. The various roles of sgRNAs in SARS-CoV-2 infection and pathogenesis 73 remain to be elucidated, but the rapid accumulation and persistence of sgRNAs following 74 infection may also contribute to disease progression.

Understanding the viral dynamics of SARS-CoV-2 and the host response are essential 77 in devising strategies to develop antiviral treatments or vaccines and curb new infections. 78 Existing PCR-based diagnostic assays for SAR-CoV-2, which are interpreted in a qualitative or semi-quantitative manner (positive, negative or indeterminate) and target only 1-2 viral regions, do not distinguish between genomic and subgenomic RNA or account for possible differences between the RNA copy numbers of various viral genes, which may depend on the degree to which they are transcribed as various sgRNAs and the degree to which the sample includes virion or cell-associated RNA. Molecular assays that can quantify different viral genes found in genomic and sgRNA species will have utility in charting the extent of viral replication and changes in SARS-CoV-2 transcription over the course of infection.

We have devised a novel panel of seven ddPCR-based assays that target various conserved regions of SARS-CoV-2 RNA, including the 5' and 3' untranslated regions, nonstructural genes that are only found in full length (genomic) RNA and structural genes that are also contained in different sgRNAs (Fig.1 and Table 1).

We selected genes encoding two non-structural proteins [Main Proteinase (NSP5) and

91 RNA dependent RNA polymerase (RdRp-NSP12)] and four major structural proteins [Spike 92 glycoprotein (S), envelope (E), membrane (M), and nucleocapsid (N)] that are known to serve 93 critical functions in SARS-CoV-2 infection. For the spike protein, in which notable mutations 94 have emerged ${ }^{22}$, we designed a primer/probe set to target the short, highly-conserved 
95

'polybasic cleavage site' ('S-PBCS') of SARS-CoV-2 which is functionally cleaved to yield the S1 and S2 subunits ${ }^{23}$, in a similar manner to the hemagglutinin (HA) protein of avian influenza viruses (AIVs) ${ }^{24}$. In AIVs, the insertion or substitution of basic amino acids at the HA cleavage site is associated with enhanced pathogenicity ${ }^{25,26}$. The SARS-CoV-2 PBCS allows effective cleavage by host furin and other proteases ${ }^{5}$, and may potentially enhance its infectivity in humans and distinguish it from related animal coronaviruses ${ }^{4,5,27}$. Elucidating the granular detail of SARS-CoV-2 transcription could help us to understand how the virus replicates and how it may evade human immune defenses. Detailed mapping of the expressed viral transcripts across times and cell types is essential for further studies of viral gene expression, mechanisms of replication, and probing host-viral interactions involved in pathogenicity.

\section{Materials and methods}

\subsection{Primer design and selection}

Multiple primer and probe sets were designed to target various regions of SAR-CoV2 , including untranslated regions that likely play an important role in regulating transcription (5' and 3' untranslated regions [UTR]), non-structural genes found only in genomic RNA (main protease [NSP5; ORF1a], RNA-dependent RNA polymerase [RdRp; ORF1b]), and structural genes that may also be found in various sgRNAs (spike [S] protein [ORF2] polybasic cleavage site [PBCS], membrane [M] glycoprotein [ORF5], and nucleocapsid [N] protein [ORF9]). Primers/probes were designed using the Primer Quest $®$ Tool (Integrated DNA Technologies, Coralville, IA). A multiple sequence alignment was performed using Clustal Omega ${ }^{28}$, encompassing complete sequences of 86 SARS-CoV-2 isolates from all geographical locations and all sequences available from the US on 3/14/2020. Reference sequences of other coronaviruses, including SARS-CoV (NC_004718.3), MERS-CoV (NC_019843.3), HCoV-229E (NC_002645.1), HcoV-NL63 (NC_005831.2), HcoV-OC43 (NC_006213.1), and HcoV-HKU1 (NC_006577.2), were included in the alignment to exclude primer sets with significant overlap with non-SARS-CoV-2 sequences. Two primer/probe sets that aligned to all SARS-CoV-2 isolates but had 1 or more mismatch with SARS-CoV and greater than 5 
123 mismatches with MERS-CoV, HCoV-229E, HCoV-NL63, HCoV-OC43, HCoV-HKU1 were

124 selected for each region (Table 1). A sequence similarity analysis using Basic Local Alignment

125 Search Tool (BLAST) ${ }^{29}$ found no significant similarity in any primer or probe to human 126 sequences.

127

\subsection{Validations using plasmid DNA}

Plasmid constructs containing the regions of interest (5'UTR, 3'UTR, Main Proteinase,

$130 \mathrm{M}$ gene, $\mathrm{N}$ gene, S protein, and a 528nt fragment of RdRp) were designed in pBluescript KS(+)

131 (Bio Basic Inc., Ontario, Canada) to enable assay validations using DNA and for use in in vitro

132 transcription reactions to generate viral RNA for standards. Plasmid concentrations were

133 quantified using ultraviolet (UV) spectrophotometry (NanoDrop ND-1000 instrument, Thermo

134 Fisher) and the molecular weights were used to calculate the number of molecules per $\mu \mathrm{L}$.

135 Extracted PBMC from a healthy donor (150-200 ng/well) and $\mathrm{H}_{2} \mathrm{O}$ were included as negative 136 controls for each assay.

137 Each primer and probe set was tested using droplet digital PCR (ddPCR), as 138 performed using the QX100 system (Bio-Rad). Droplet digital PCR was chosen because it enables "absolute" quantification, it is relatively less dependent on PCR efficiency (which may

140 be reduced by sequence mismatches or inhibitors), and it may be more precise than 141 quantitative PCR (qPCR) at low copy numbers ${ }^{30}$. Plasmid DNA was added to ddPCR wells at 142 expected inputs of $1-10^{3}$ copies/well in duplicate (1000 and 100 copies) or quadruplicate (10 143 and 1 copy). Each reaction consisted of $20 \mu \mathrm{L}$ per well containing $10 \mu \mathrm{L}$ of ddPCR Probe 144 Supermix (no deoxyuridine triphosphate), $900 \mathrm{nM}$ of primers, $250 \mathrm{nM}$ of probe, and $5 \mu \mathrm{L}$ of 145 plasmid DNA. Droplets were amplified using a Mastercycler® nexus (Eppendorf, Hamburg, 146 Germany) with the following cycling conditions: $10 \mathrm{~min}$ at $95^{\circ} \mathrm{C}, 45$ cycles of $30 \mathrm{~s}$ at $95^{\circ} \mathrm{C}$ and $14759^{\circ} \mathrm{C}$ for $60 \mathrm{~s}$, and a final droplet cure step of $10 \mathrm{~min}$ at $98^{\circ} \mathrm{C}$. Droplets were read and analyzed 148 using the QuantaSoft software in the absolute quantification mode. 


\subsection{Validations using synthetic RNA}

In vitro transcribed (IVT) RNA standards were generated from the aforementioned

152 plasmids using the T7 RiboMAX ${ }^{\text {TM }}$ Express Large-Scale RNA Production System (Promega,

153 Madison, WI). The concentration of each IVT RNA standard was measured by Nanodrop and

154 the molecular weight was used to calculate the expected number of molecules per $\mu \mathrm{L}$. The

155 length, integrity, and concentration of each IVT standard were confirmed using the Agilent

156 Bioanalyzer RNA 6000 Nano assay (Agilent, Santa Clara, CA) prior to dilution in nuclease-

157 free water to working concentrations.

158 A reverse transcription (RT) reaction was performed in $50 \mu \mathrm{L}$ containing $5 \mu \mathrm{L}$ of $10 \times$

159 SuperScript III buffer (Invitrogen), $5 \mu \mathrm{L}$ of $50 \mathrm{mM} \mathrm{MgCl}$, $2.5 \mu \mathrm{L}$ of random hexamers (50 $160 \mathrm{ng} / \mu \mathrm{L}$; Invitrogen), $2.5 \mu \mathrm{L}$ of $50 \mu \mathrm{M}$ poly-dT15, $2.5 \mu \mathrm{L}$ of $10 \mathrm{mM}$ deoxynucleoside triphosphates

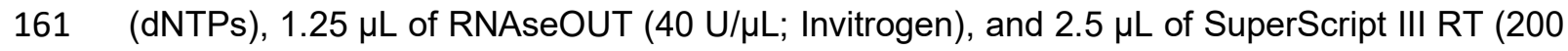

$162 \mathrm{U} / \mu \mathrm{L}$; Invitrogen). Although the IVT standards were not polyadenylated, reverse transcription

163 was performed with both random hexamers and poly-dT because we anticipated that these

164 assays would be applied to clinical samples containing long polyadenylated SARS-CoV-2

165 RNAs, for which the combination of poly-dT plus random hexamers may reduce bias towards reverse transcription of any one region (as can be seen with specific reverse primers), the 5'

167 end (as would be expected with random hexamers), or the 3' end (as would be expected with 168 poly-dT).

169 IVT RNA standards were added to RT reactions at inputs of $1,10,10^{2}, 10^{3}$, and $10^{4}$ 170 copies per $5 \mu \mathrm{L}$ (2 replicate RT reactions for each input). RT reactions were performed in a 171 conventional thermocycler at $25.0^{\circ} \mathrm{C}$ for $10 \mathrm{~min}, 50.0^{\circ} \mathrm{C}$ for $50 \mathrm{~min}$, followed by an inactivation 172 step at $85.0^{\circ} \mathrm{C}$ for 5 min. Undiluted RT product $(5 \mu \mathrm{L})$ was added to ddPCR reactions (total 173 volume of $20 \mu \mathrm{L}$ ) and ddPCR was performed as described for 'Validations using plasmid DNA'.

174 Primer-probe sets for each target region were tested head-to-head using this approach. Based 175 on performance of each primer-probe set using plasmid DNA and IVT RNA, one primer/probe 176 set for each region was selected for further testing. 
177 To determine the robustness of our approach, in addition to testing each assay with

178 varying RNA copy inputs (each with two replicate RT reactions per input and replicate ddPCR

179 wells for each RT), we performed repeat, independent experiments using the same 180 parameters to confirm each assay's efficiency and sensitivity ( $n=4$ for N-ORF9, CDC_N1, and

181 CDC_N2; n=3 for 5'UTR, 3'UTR; and n= 2 for all others). No data were excluded as outliers.

\subsection{Validations using SARS-CoV-2 virion RNA}

Vero CCL-81 kidney epithelial cells, derived from Cercopithecus aethiops, were

infected with SARS-CoV-2 (Isolate: USA-WA1/2020) at an MOI of 0.003 (250 000 cells/well).

Cells were incubated for 72 hours at $37^{\circ} \mathrm{C} / 5 \% \mathrm{CO}_{2}$ and harvested. Viral supernatant was clarified by 2 centrifugation steps (180 $\mathrm{xg}, 5 \mathrm{~min}$ ) and added directly to $1 \mathrm{~mL}$ TRI reagent

(Molecular Research Center Inc.). Total RNA was extracted using TRI reagent, including the addition of polyacryl carrier $(2.5 \mu \mathrm{L})$. Extracted RNA was then subjected to two rounds of

DNase I treatment as follows to ensure degradation and removal of contaminating DNA. First, incubated at $37^{\circ} \mathrm{C}$ for 15 minutes. Next, virion RNA was purified using the RNeasy Mini Kit with on-column DNase digestion with RNase-Free DNase I (Qiagen). The copies/ $\mu \mathrm{L}$ in the virion standard were estimated by triplicate measurements using the Abbott RealTime SARSCoV-2 assay (Abbott m2000 Molecular Platform). Dilutions of the virion standard were added to $\mathrm{RT}$ reactions to achieve expected inputs of 1 to 70,000 copies per $5 \mathrm{uL}$ RT (the input into each ddPCR well). RT reactions were performed as above, with random hexamers and polycDNA could then be used to test each assay in parallel using replicate $20 \mathrm{uL}$ ddPCR reactions

201 (see above) containing primers/probe specific for a given region. 
204 Further validations were performed to determine each assay's sensitivity to inhibition

205 by "background" cellular RNA, as would be expected in clinical samples containing cells. The

206 virion standard (1000 copies per $5 \mu \mathrm{L}$ RT) was added to RT reactions with or without cellular

207 RNA from A549 cells (lung epithelial cell line) or donor PBMC (both added at 100ng/ $\mu$ l per RT,

208 or 500 ng per ddPCR well). RT reactions contained a total of $125 \mu \mathrm{L}$ with $12.5 \mu \mathrm{L}$ of $10 \times$

209 SuperScript III buffer (Invitrogen), $12.5 \mu \mathrm{L}$ of $50 \mathrm{mM} \mathrm{MgCl}$, $6.25 \mu \mathrm{L}$ of random hexamers (50

$210 \mathrm{ng} / \mu \mathrm{L}$; Invitrogen), $6.25 \mu \mathrm{L}$ of $50 \mu \mathrm{M}$ dT15, $6.25 \mu \mathrm{L}$ of $10 \mathrm{mM}$ deoxynucleoside triphosphates

211 (dNTPs), $3.125 \mu \mathrm{L}$ of RNAseOUT (40 U/ $\mu \mathrm{L}$; Invitrogen), and $6.25 \mu \mathrm{L}$ of SuperScript III RT (200

$212 \mathrm{U} / \mu \mathrm{L}$; Invitrogen). $\mathrm{RT}$ reactions were incubated at $25.0^{\circ} \mathrm{C}$ for $10 \mathrm{~min}, 50.0^{\circ} \mathrm{C}$ for $50 \mathrm{~min}$,

213 followed by an inactivation step at $85.0^{\circ} \mathrm{C}$ for $5 \mathrm{~min}$. Undiluted cDNA ( $5 \mu \mathrm{L}$ ) was added to each

$21420 \mu \mathrm{L}$ ddPCR reaction and replicate ddPCR reactions were performed for each assay.

\subsection{Assay validations in clinical diagnostic samples from SARS-CoV-2 infected}

\section{7 individuals}

218 To investigate the viral transcription profile in clinical samples and determine whether our RT-ddPCR assays correlate with a clinical test, we obtained unused nucleic acid (ranging from $8.25-16.8 \mu \mathrm{L}$ ) that remained after extraction by the Abbott $\mathrm{m} 2000$ instrument from nasopharyngeal swabs from 3 individuals who tested positive with the Abbott Real Time SARS-CoV-2 assay. Nucleic acid from these 3 individuals, who had $C_{t}$ values of $11.59,15.81$, and 19.14 (respectively) on the Abbot assay, was tested using our RT-ddPCR assays for the 5'UTR, Main Proteinase, RdRp, S, M, N and 3'UTR regions. The available volume of nucleic acid was added into $85 \mu \mathrm{L}$ RT reactions containing $1 \times$ SuperScript III buffer, $5 \mathrm{mM} \mathrm{MgCl}$, 2.5 ng of random hexamers, $2.5 \mu \mathrm{M}$ dT15, $0.5 \mathrm{mM}$ deoxynucleoside triphosphates (dNTPs), $1 \mathrm{U} / \mu \mathrm{L}$ of RNAseOUT, and $10 \mathrm{U} / \mu \mathrm{L}$ of SuperScript III RT. RT reactions were performed under the aforementioned conditions. Undiluted cDNA was divided evenly across assays $(5 \mu \mathrm{L}$ input into each ddPCR well, tested in duplicate) and ddPCR reactions were performed under the 
231 ddPCR were adjusted to account for differing input volume of nucleic acid to yield the SARS-

232 CoV-2 copies/ $\mu \mathrm{L}$ extract. The log-linear relationship between viral load measured by RT-PCR

233 (Abbott Real Time SARS-CoV-2 assay) and RT-ddPCR was determined using GraphPad

234 Prism (version 8.4.1).

\section{Results}

\subsection{Detection limit, linearity, and efficiency using plasmid DNA}

Two assays were designed for each region (indicated in Fig. 1; 'Assay locations')

243 plasmid is technically challenging (due to the $30 \mathrm{~kb}$ length) and subject to higher biosafety restrictions, we constructed or purchased plasmids containing individual genes or regions.

the number of molecules (expected copies) was calculated using the molecular weight.

247 Each assay was assessed for detection limit, dynamic range, linearity, and efficiency by

248 measuring the absolute number of copies detected using droplet digital PCR (ddPCR) from

249 expected inputs of serially diluted plasmid DNA. All assays could detect as few as 1-10 copies

250 and were linear over at least 3 orders of magnitude $\left(R^{2}>0.99\right.$ for all; Fig. 2$)$. Assay efficiencies

251 (measured by the slope) varied somewhat between assays, ranging from 0.67 ("N-ORF9_8")

252 to 1.1 (“M-ORF5"). One assay from each pair was selected for further study (Table 2; rejected

253 primer/probe sets are listed in Table S1) based on the overall efficiency (Fig. 2), separation

254 between the positive and negative droplets [amplitude/signal to noise] (Fig. S2), and specificity

255 (Table S2). For the chosen assays, no positive droplets were detected with water or DNA

256 from peripheral mononuclear blood cells (PBMC) from uninfected blood donors. 


\subsection{Detection limit, linearity, and efficiency using in vitro transcribed and virion RNA}

Selected assays for each region were tested using standards prepared from in vitro Bioanalyzer) and the molecular weight. Using RT-ddPCR, all assays could detect as few as 10 copies of RNA and demonstrated linearity over $3-4$ orders of magnitude $\left(R^{2}>0.999\right.$ for all;

Fig. 3). The efficiencies for detecting IVT RNA standards, which ranged from 0.18 (for Main Proteinase) to 0.96 (S-PBCS), were more variable than those observed for plasmid DNA. No amplification was detected in 'No RT' control reactions containing 10,000 IVT RNA copies/well, confirming the absence of any contaminating plasmid DNA. However, it is worth noting that none of these IVT standards were polyadenylated (so they should not be reversetranscribed by poly-dT) and some of the standards were very short ( $<300$ base pairs), which would likely limit the efficiency with which they were reverse transcribed by random hexamers.

273 In addition, some of the measured differences in efficiency could reflect actual differences in

274 the copy numbers present in the various IVT standards, which are difficult to determine precisely.

To circumvent these limitations, we prepared one SARS-CoV-2 'virion' standard containing all of the target regions by extracting RNA from cell-free supernatant from a cell

279 line (Vero CCL81) infected in vitro with a SARS-CoV-2 patient isolate (USA-WA1/2020). The

280 expected copies in this virion standard were calculated using the $C_{t}$ value measured by the 281 Abbott m2000 Real Time SARS-CoV-2 viral load assay, which targets the $\mathrm{N}$ and RdRp genes 282 using probes labelled with the same fluorophore. This virion standard enabled the preparation 283 of common RT reactions containing specific inputs of SARS-CoV-2 genomic equivalents, from which aliquots of cDNA could be divided evenly across our panel of assays for simultaneous assessment of all target regions in ddPCR reactions (Fig. 4). 
Expected inputs of 10 to 70,000 copies per well were used to measure the absolute

copies of 5'UTR, Main Proteinase, RdRp, S, M, N and 3'UTR regions. All assays detected as

few as 10 copies of the virion standard and were linear over four orders of magnitude

289 ( $R^{2}>0.999$ for all). No amplification was detected in 'No RT' control reactions containing 10,000

290 IVT RNA copies/well. Assay efficiencies were all greater than 1.0 (range: 1.05 to 2.46), likely

291 because the estimate from the Abbott assay was lower than the true value and/or the RT-

292 ddPCR assays are more efficient. In addition, the efficiency of the RT-ddPCR assays

293 increased from 5' to 3' targets, which could reflect the presence of 3' subgenomic RNAs in the

294 virion standard or greater efficiency of reverse transcription from the 3' end of the genome.

295

\subsection{Assay specificity and false positive rate}

To determine the non-specific reactivity of oligonucleotides (false positive rate) for each assay, we performed a median of 26 [range 18-32] 'no template' controls (NTC). These

reactions were performed with both water (water NTC) and DNA or RNA isolated from SARS-

CoV-2-negative donor PBMC (DNA/RNA NTC) (Table S2). Except for one experiment using

IVT RNA, where a total of three droplets were detected across duplicate NTC wells containing donor PBMC tested for Main Proteinase-NSP5, no other false positives were observed.

\subsection{Comparison of new and existing SARS-CoV-2 assays in ddPCR platform}

Our assay panel included new primers/probes for the nucleocapsid (N-ORF9), which

is targeted by existing diagnostic real-time PCR assays. We compared the performance of our

307 'N-ORF9' primers/probe to the primers/probes from the U.S. Center for Disease Control assays for the nucleocapsid (CDC-N1 and CDC-N2) ${ }^{31}$ using ddPCR. The N-ORF9 assay efficiency was similar to that of CDC-N1 and CDC-N2 for plasmid DNA, in between that of CDC-N1 and CDC-N2 for IVT RNA, and similar to CDC-N1 for the virion standard (Fig. 2-4).

312 for the "IP2" assay ${ }^{32}$ (which targets ORF1a) and "E-Sarbeco" 33 assay (which targets the E 313 gene) using RT-ddPCR and the virion standard (Fig. 5; Table 3). The IP2 (ORF1a) assay 
314 efficiency was 1.11, compared to $1.20-1.28$ for our RdRp (ORF1b) and 1.36 for our main

315 protease (ORF1a) assays (Fig. 4-5). The E-Sarbeco [ORF4] assay efficiency (1.08) was

316 similar to the IP2, but may have been less than our assays targeting neighboring genes (S-

317 PBCS [ORF2]: 1.32; M-ORF5: 1.51).

318

319

320

321

322

323

324

325

326

327

328

329

330

331

332

\subsection{Lower limit of detection of SARS-CoV-2 in RNA}

Our validation studies included SARS-CoV-2 RNA inputs down to 1 copy per ddPCR reaction (Fig. 2-3). We estimated the lower limit of detection (LLOD) for each assay in our panel based on data for all replicates tested at 10 copy and 1 copy inputs (Table S3). At 10 copies, all of our assays detected SARS-CoV-2 in $\geq 85.7 \%$ of tests (range $=85.7-100 \%$ ). At 1 copy input, our assays detected SARS-CoV-2 in $\geq 25 \%$ of tests (range $=25-88 \%$ ), underscoring the high sensitivity of our assays.

\subsection{Effect of Background RNA on assay efficiencies}

Next, we assessed the efficiencies of our assays in the presence of "background" RNA from uninfected cells (Fig. 6). At a constant input of 1000 copies of the SARS-CoV-2 virion RNA, we determined the effect of adding cellular RNA (100ng per $\mu \mathrm{L}$ of RT) extracted from PBMC or a lung epithelial cell line (A549 cells). All assays showed slightly greater efficiency in the presence of $100 \mathrm{ng} / \mu \mathrm{L}$ background RNA from either PBMC or A549 cells compared to the virion standard with no background RNA. No false positives were detected with $100 \mathrm{ng} / \mu \mathrm{L}$ RT RNA from PBMC, while 1-4 droplets were sometimes detected in the RNA from A549 cells using some assays (Main Proteinase, RdRp, S-PBCS). Overall, these data suggest that in samples derived from individuals infected with SARS-CoV-2, our assays are likely to be minimally inhibited by background RNA, making them ideally suited to a diverse range of clinical samples.

\subsection{Strong correlation between viral loads measured by RT-ddPCR and real-time PCR} in clinical diagnostic samples 
To compare our assays with a clinical test, we obtained unused nucleic acid that had

344 been extracted by the Abbott m2000 molecular platform from nasopharyngeal swabs from

345 three SARS-CoV-2-infected individuals and remained after clinical testing using the Abbott

346 Real Time SARS-CoV-2 assay. Using this nucleic acid, we measured RNA levels of the

347 5'UTR, Main Proteinase, RdRp, S, M, N and 3'UTR regions using our RT-ddPCR assays. (Fig.

348 7). As observed with the virion standard, transcripts containing the most 3' regions (N-ORF9

349 and 3 'LTR) tended to be present at higher copy numbers, while those containing the 5'LTR

350 tended to be present at lower levels. However, the order of transcript levels varied somewhat

351 between individuals and sometimes differed from the 3' to 5' gradient observed with the virion

352 standard. For example, levels of S-PBCS RNA tended to be lower than those of the more 5'

353 Main Protease (NSP5) transcripts. These potential differences in SARS-CoV-2 transcription

354 profile may reflect changes in viral dynamics over the course of infection or inter-individual

355 variability in viral sequences or host responses, and should be confirmed in future studies

356 using longitudinal samples from more individuals.

Next, we determined the correlation between the $C_{t}$ value as measured by the Abbott assay and SARS-CoV-2 copy numbers as determined by RT-ddPCR. For each target, this relationship was modelled using linear regression following log transformation of SARS-CoV2 copies/ $\mu \mathrm{L}$ extract [where $y=\log _{2}(x)$ ] (Fig S1). The coefficient of determination $\left(R^{2}\right)$ for each

362 model was $\geq 0.93$ for all targets, underscoring the log-linear relationship between ddPCR-

363 based SARS-CoV-2 transcript levels and $C_{t}$ values in diagnostic specimens. Taken together,

364 these data strongly underscore the sensitivity of our assays, demonstrating the ability to detect

365 all targets using minimal RNA inputs (effectively 1.2-2.4 $\mu \mathrm{L}$ RNA input per assay), and their

366 strong correlation with $\mathrm{C}_{\mathrm{t}}$ values obtained by real-time PCR using clinical assays. Furthermore,

367 these data highlight that delineation of the SARS-CoV-2 transcription profile in samples across

368 differing timepoints within and between participants may yield valuable insight into viral

369 transcription dynamics across the course of SARS-CoV-2 infection. 


\section{4. Discussion}

372 The 2019 SARS-CoV-2 outbreak has heralded the development of an array of

373 diagnostic molecular tools to study this novel coronavirus. However, currently described PCR-

374 based diagnostic assays are qualitative or semi-quantitative, are limited to the simultaneous

375 detection of one or two regions, and do not distinguish genomic from subgenomic RNAs.

376 Here, we report a panel of new primer/probe sets that span the SARS-CoV-2 genome and

377 target important nongenic regions, non-structural genes found only in genomic RNA, and

378 structural genes that are also found in different subgenomic RNAs.

379 We used these new primers/probes for RT-ddPCR rather than qRT-PCR because 380 ddPCR provides absolute quantification (does not require an external calibrator), tends to

381 tolerate sequence mismatches in primer/probe sequences better than qRT-PCR, and may be

382 more precise at low copies, while providing similar sensitivity and reproducibility ${ }^{30,35}$. During

383 validation of these assays with multiple different standards, we sometimes found that the

384 efficiency of the same assay varied somewhat across different standards. These differences

385 may reflect differences in the nature of the standards (DNA, short in vitro transcribed non-

386 polyadenylated RNA, or "virion RNA") as well as the difficulty in determining the exact number

387 of copies in an external standard; the latter issue highlights a major advantage of the absolute

388 quantification provided by ddPCR. On all standards tested, the seven RT-ddPCR assays were

389 extremely sensitive (down to 1-10 copies) and linear over 3-4 orders of magnitude, and all

390 seven assays showed no inhibition by up to 500,000 cell equivalents of RNA per ddPCR well,

391 suggesting that these assays could be extremely useful for SARS-CoV-2 research. While most

392 existing clinical assays for SARS-CoV-2 use qPCR because it is less expensive and may have

393 fewer false positives than ddPCR, it is likely that the primer/probe sets described here would

394 also work well in qPCR assays for research or clinical testing.

395 The utility of assays that target multiple genomic regions is supported by studies

396 demonstrating loss in sensitivity of published assays owing to mutations that could affect

397 primer annealing. For instance, a recent study found that $34.38 \%(11,627)$ of SARS-CoV-2

398 genomes featured a single mutation capable of affecting annealing of a PCR primer in tested 
assays from the World Health Organization, Centers for Disease Control and Prevention,

400 National MicrobiologyData Center, and Hong Kong University ${ }^{36}$. Another study found single

401 nucleotide mismatches in $0.2 \%$ and $0.4 \%$ of the surveyed SARS-CoV-2 sequences compared

402 to the CDC-N1 probe and reverse primer, respectively, and $0.4 \%$ of those sequences

403 compared to Charité's E_Sarbeco_R primer ${ }^{34}$. Therefore, a strategy that can target multiple

404 genomic regions may have utility in sensitive detection of SARS-CoV-2.

405 Extensive, well-designed studies have assessed the analytical sensitivity and 406 efficiency of existing RT-qPCR primer-probes sets ${ }^{34,37-39}$ and explored adaptation of such 407 assays to the ddPCR platform ${ }^{40}$. In this study, we describe how some of the available 408 diagnostic assays compare to our novel SARS-CoV-2 assays and report how a multi-assay 409 approach using the ddPCR platform could significantly advance our understanding of SARS-

410 CoV-2 transcription and replication. While highly-sensitive PCR-based assays might not be 411 essential to identify SARS-CoV-2-infected individuals in the transmissible/contagious phase 412 of infection, quantitative assays capable of detecting very low copies of SARS-CoV-2 will be 413 particularly useful in understanding the course of infection and correlates of disease 414 progression. Existing clinical assays are quite sensitive for detecting COVID-19 during the first 415 several weeks of infection, but often become negative after 2-3 weeks of infection ${ }^{41-43}$. In 416 some cases, individuals who test positive may have a subsequent negative test followed by 417 another positive or alternating positive and negative tests ${ }^{44,45}$. Some individuals may also 418 have prolonged viral shedding after symptomatic relief, with one study noting a patient with 419 qRT-PCR positivity detected in upper respiratory tract samples 83 days post-symptom onset ${ }^{46}$. 420 Therefore, sensitive assays such as those described in the study could be of great utility in 421 studying the course of infection two or more weeks after the resolution of acute symptoms. 422 Another advantage of the approach described here is that it permits a single sample to be 423 simultaneously assayed for multiple targets, which may increase sensitivity and specificity 424 while helping to delineate the transcriptional profile of SARS-CoV-2 in infected patient 425 samples. As such, this panel of assays can be applied to a diverse range of clinically relevant 426 samples in which SARS-CoV-2 RNA may be in low or high abundance. 
427 Using both the virion standard and clinical samples from the nasopharynx, we tended

428 to observe higher copy numbers for targets at the 3' end of the genome (N, 3'UTR) compared

429 to the 5' end (5'UTR, main protease). This discrepancy is not explained by differences in PCR

430 efficiency, since the efficiency of the $\mathrm{N}$ assay on plasmid DNA was actually lower than that of

431 assays for the 5'UTR or main protease. It is possible that reverse transcription is more efficient

432 for assays at the 3' end (perhaps due to more efficient reverse transcription from the poly-dT),

433 although random hexamers should bias towards the 5' end and the combination has been

434 used to prevent bias towards either the $5^{\prime}$ or $3^{\prime}$ end of the $9.6 \mathrm{~kb}$ genome of HIV-1 ${ }^{30,47}$. It is also

435 possible that the 3 ' assays measure higher copies because they are detecting subgenomic

436 RNAs generated by infected cells and not packaged into virions ${ }^{48}$, which may have been

437 present in the supernatant used to prepare the virion standard if they were released from dying

438 cells or present in low levels in exosomes. This excess of targets corresponding to sgRNAs

439 may be much greater in samples that contain more cells or cell-associated RNA, and it has

440 important implications for clinical testing and research. For targets in the 3' third of the genome

441 that are transcribed as sgRNAs, regions that are further downstream (3') may be incorporated

442 into a greater variety of sgRNAs and therefore should be present at higher copy numbers, so

443 assays targeting these regions may be more sensitive to detect infection ${ }^{8}$. On the other hand,

444 sgRNAs are not infectious, so assays targeting more 5' regions that are transcribed only as

445 genomic RNA (ORF 1a and 1b) may correlate better with infectivity.

446 The clinical implications of SARS-CoV-2 subgenomic RNA transcription are currently

447 unknown. The synthesis of subgenomic RNAs is a common strategy employed by positive-

448 sense RNA viruses to transcribe their 3' proximal genes that encode products essential for

449 particle formation and pathogenesis ${ }^{49-51}$. In coronaviruses such as mouse hepatitis virus

450 (MHV), the synthesis of subgenomic RNAs may function as important mediators of positive

451 strand synthesis ${ }^{52}$, and more broadly, members of the order Nidovirales (including

452 Coronaviridae) feature high levels of redundancy to ensure continued protein synthesis even

453 in the event of point mutations in regulatory sequences ${ }^{53}$. The characterization of the SARS-

454 CoV-2 transcription profile in differing patient samples over the course of infection may provide 
455 insight into the molecular mechanisms by which SARS-CoV-2 regulates gene expression 456 through differential transcription of genomic and subgenomic RNAs, and how this differential 457 gene expression may contribute to pathogenesis.

458 We found that our assays performed better in the presence of background RNA, 459 irrespective of origin (blood or epithelial cells, Fig. 6). This finding accords with other studies 460 that have extensively validated the effect of differing variables on RT efficiency and suggest 461 that the presence of some background RNA may increase efficiency of the reverse 462 transcription step ${ }^{54-56}$. While the efficiency of our assays tended to decrease with RNA 463 concentrations above $100 \mathrm{ng} / \mu \mathrm{L} \mathrm{RT}$, even at 500ng RNA/ $\mu \mathrm{L} \mathrm{RT}$, these assays still performed 464 better than in the absence of any background RNA, suggesting that they are ideally suited for 465 testing samples from different tissues where the levels of genomic RNA may differ 466 considerably. Furthermore, our comparison of viral loads obtained by RT-ddPCR and qRT467 PCR demonstrates the strong correlation between data obtained from these two platforms and the minimal RNA input required to yield robust data using our RT-ddPCR assays. with published assays (total of 11 assays) in background RNA experiments (Fig. 7), we 471 increased RT reaction volumes from $50-70 \mu \mathrm{L}$ to $125 \mu \mathrm{L}$ to accommodate the additional 472 assays. In the absence of background RNA, the efficiency appeared to be higher in the 50$47370 \mu \mathrm{L}$ RT reactions (Fig. 4-5, >100\% efficiency for all assays) than the $125 \mu \mathrm{L}$ reactions (Fig. 4747 ; median efficiency $=88 \%$ [range: $60-133 \%$ ]). If the discrepancy is not due to a difference in 475 the actual input of the standard, it is possible that larger reaction volumes lead to less 476 efficiency in reverse transcription. However, for application to patient samples, our core panel 477 of 7 assays (Table 1 ) is sufficient to provide a detailed view of the transcription profile of SARS478 CoV-2, so preparation of RT reactions $>70 \mu \mathrm{L}$ will likely be unnecessary.

479 For our study of the viral transcription profile and correlation with the $\mathrm{C}_{\mathrm{t}}$ value as 480 determined by the Abbott SARS-CoV-2 Real Time Assay, a limited amount of nucleic acid was available from only a small number of de-identified individuals. Despite this small sample 482 size, we demonstrated both the sensitivity of all assays in our panel and their strong correlation 
483 with $C_{t}$ values in diagnostic specimens. These data allude to potential differences in the

484 transcription dynamics of SAR-CoV-2 during the course of infection and merit further

485 investigation.

486

\section{Conclusions}

488 We developed a panel of sensitive, quantitative RT-ddPCR-based SARS-CoV-2 assays

489 that collectively span the genome and target nongenic and genic regions, genes encoding for

490 important enzymes and structural proteins, and genes found in different subgenomic RNAs.

491 These assays can serve as novel molecular tools to investigate SARS-CoV-2 infection,

492 replication dynamics, and gene expression to better understand the viral dynamics and

493 pathogenesis of SARS-CoV-2 over the course of infection. Future studies employing these

494 assays will enhance our understanding of SARS-CoV-2 replication and transcription and may

495 also inform the development of improved diagnostic tools and therapeutics. 
497 Additional Information:

498 Funding:

499 This research was supported by funds from the Emergency COVID-19 Research Seed 500 Funding of the University of California (Grant Number R00RG3113 [ST]). The investigators 501 received salary support from the U.S. Department of Veterans Affairs (SAY and JKW), the

502 National Institute of Diabetes and Digestive and Kidney Diseases at the NIH (R01DK108349 503 [SAY, JKW], R01DK120387 [SAY]), the National Institute of Allergy and Infectious Diseases 504 at the NIH (R01Al132128 [SAY, JKW]), the UCSF/GIVI Center for AIDS Research (CFAR; 505 Grant\# P30 Al027763 [ST]; award \#A120163 [PI: Paul Volberding]), and the California 506 HIVIAIDS Research Program (Grant number BB19-SF-009 [ST]). The funders had no role in 507 study design, data collection and analysis, decision to publish, or preparation of the 508 manuscript.

$510 \quad$ Ethics

511 This study included the use of de-identified nucleic acid from three SARS-CoV-2512 infected individuals. The study authors had no subject contact or access to any personally513 identifiable information (Category 4, IRB exempt).

\section{CRediT authorship contribution statement}

516 Sushama Telwatte: Conceptualization, Data curation, Formal analysis, Funding acquisition, 517 Investigation, Methodology, Supervision, Validation, Visualization, Writing - original draft.

518 Nitasha Kumar: Investigation, Writing - review \& editing. Chuanyi M. Lu: Resources, Writing 519 - review \& editing. Alberto Vallejo-Gracia: Investigation, Writing - review \& editing. G. 520 Renuka Kumar: Investigation, Writing - review \& editing. Melanie Ott: Resources, 521 Supervision, Writing - review \& editing. Joseph K. Wong: Resources, Supervision, Writing 522 review \& editing. Steven A. Yukl: Conceptualization, Funding acquisition, Methodology, 523 Resources, Supervision, Writing - original draft. 
bioRxiv preprint doi: https://doi.org/10.1101/2021.01.12.425991; this version posted January 12, 2021. The copyright holder for this preprint (which was not certified by peer review) is the author/funder, who has granted bioRxiv a license to display the preprint in perpetuity. It is made available under aCC-BY-NC-ND 4.0 International license.

\section{Conflicts of interest statement}

526 The authors declare that they have no competing interests.

527 


\section{References}

529 1. Zhu N, Zhang D, Wang W, et al. A Novel Coronavirus from Patients with Pneumonia 530 in China, 2019. N Engl J Med 2020;382:727-33.

5312 Kim JM, Chung YS, Jo HJ, et al. Identification of Coronavirus Isolated from a Patient in 532 Korea with COVID-19. Osong Public Health Res Perspect 2020;11:3-7.

533 3. Lu R, Zhao X, Li J, et al. Genomic characterisation and epidemiology of 2019 novel 534 coronavirus: implications for virus origins and receptor binding. Lancet 2020;395:565-74.

535 4. Zhou H, Chen X, Hu T, et al. A Novel Bat Coronavirus Closely Related to SARS-CoV-2

536 Contains Natural Insertions at the S1/S2 Cleavage Site of the Spike Protein. Curr Biol 537 2020;30:2196-203.e3.

538 5. Andersen KG, Rambaut A, Lipkin WI, Holmes EC, Garry RF. The proximal origin of 539 SARS-CoV-2. Nat Med 2020;26:450-2.

540 6. Malaiyan J, Arumugam S, Mohan K, Radhakrishnan GG. An update on origin of SARS541 CoV-2: Despite closest identity, bat (RaTG13) and Pangolin derived Coronaviruses varied in 542 the critical binding site and O-linked glycan residues. J Med Virol 2020.

5437 7. Sawicki SG, Sawicki DL, Siddell SG. A Contemporary View of Coronavirus 544 Transcription. J Virol 2007;81:20.

545 8. Kim D, Lee JY, Yang JS, Kim JW, Kim VN, Chang H. The Architecture of SARS-CoV-2 546 Transcriptome. Cell 2020;181:914-21.e10.

547 9. Sola I, Almazán F, Zúñiga S, Enjuanes L. Continuous and Discontinuous RNA Synthesis 548 in Coronaviruses. Annu Rev Virol 2015;2:265-88.

549 10. Snijder EJ, Limpens R, de Wilde AH, et al. A unifying structural and functional model 550 of the coronavirus replication organelle: Tracking down RNA synthesis. PLoS Biol 551 2020;18:e3000715.

552 11. Wu F, Zhao S, Yu B, et al. A new coronavirus associated with human respiratory 553 disease in China. Nature 2020;579:265-9.

554 12. Sethna PB, Hofmann MA, Brian DA. Minus-strand copies of replicating coronavirus 555 mRNAs contain antileaders. J Virol 1991;65:320-5.

556 13. Sawicki SG, Sawicki DL. Coronaviruses use discontinuous extension for synthesis of 557 subgenome-length negative strands. Adv Exp Med Biol 1995;380:499-506.

558 14. Woo PCY, Lau SKP, Chu C-m, et al. Characterization and complete genome sequence 559 of a novel coronavirus, coronavirus HKU1, from patients with pneumonia. J Virol $560 \quad 2005 ; 79: 884-95$.

561 15. Cowley TJ, Long SY, Weiss SR. The murine coronavirus nucleocapsid gene is a 562 determinant of virulence. J Virol 2010;84:1752-63.

563 16. Kopecky-Bromberg SA, Martínez-Sobrido L, Frieman M, Baric RA, Palese P. Severe 564 acute respiratory syndrome coronavirus open reading frame (ORF) 3b, ORF 6, and 565 nucleocapsid proteins function as interferon antagonists. J Virol 2007;81:548-57.

566 17. Huang C, Lokugamage KG, Rozovics JM, Narayanan K, Semler BL, Makino S. SARS 567 coronavirus nsp1 protein induces template-dependent endonucleolytic cleavage of mRNAs: 568 viral mRNAs are resistant to nsp1-induced RNA cleavage. PLoS Pathog 2011;7:e1002433.

569 18. Kamitani W, Narayanan K, Huang C, et al. Severe acute respiratory syndrome 570 coronavirus nsp1 protein suppresses host gene expression by promoting host mRNA 571 degradation. Proceedings of the National Academy of Sciences of the United States of 572 America 2006;103:12885-90. 
573 19. Narayanan K, Huang C, Lokugamage K, et al. Severe acute respiratory syndrome

574 coronavirus nsp1 suppresses host gene expression, including that of type I interferon, in

575 infected cells. J Virol 2008;82:4471-9.

576 20. Kamitani W, Huang C, Narayanan K, Lokugamage KG, Makino S. A two-pronged

577 strategy to suppress host protein synthesis by SARS coronavirus Nsp1 protein. Nat Struct

578 Mol Biol 2009;16:1134-40.

579 21. Sztuba-Solińska J, Stollar V, Bujarski JJ. Subgenomic messenger RNAs: mastering

580 regulation of (+)-strand RNA virus life cycle. Virology 2011;412:245-55.

581 22. Korber B, Fischer WM, Gnanakaran S, et al. Tracking Changes in SARS-CoV-2 Spike:

582 Evidence that D614G Increases Infectivity of the COVID-19 Virus. Cell 2020;182:812-27.e19.

583 23. Walls AC, Park YJ, Tortorici MA, Wall A, McGuire AT, Veesler D. Structure, Function,

584 and Antigenicity of the SARS-CoV-2 Spike Glycoprotein. Cell 2020;181:281-92.e6.

585 24. Böttcher-Friebertshäuser E, Garten W, Matrosovich M, Klenk HD. The hemagglutinin:

586 a determinant of pathogenicity. Curr Top Microbiol Immunol 2014;385:3-34.

587 25. Monne I, Fusaro A, Nelson MI, et al. Emergence of a highly pathogenic avian

588 influenza virus from a low-pathogenic progenitor. J Virol 2014;88:4375-88.

589 26. Horimoto T, Rivera E, Pearson J, et al. Origin and molecular changes associated with

590 emergence of a highly pathogenic H5N2 influenza virus in Mexico. Virology 1995;213:223-

59130.

592 27. Nao N, Yamagishi J, Miyamoto H, et al. Genetic Predisposition To Acquire a Polybasic

593 Cleavage Site for Highly Pathogenic Avian Influenza Virus Hemagglutinin. mBio 2017;8.

594 28. Madeira F, Park YM, Lee J, et al. The EMBL-EBI search and sequence analysis tools

595 APIs in 2019. Nucleic Acids Res 2019;47:W636-W41.

596 29. Altschul SF, Madden TL, Schäffer AA, et al. Gapped BLAST and PSI-BLAST: a new

597 generation of protein database search programs. Nucleic Acids Res 1997;25:3389-402.

598 30. Yukl SA, Kaiser P, Kim P, et al. HIV latency in isolated patient CD4(+) T cells may be

599 due to blocks in HIV transcriptional elongation, completion, and splicing. Sci Transl Med

600 2018;10.

601 31. 2019-Novel Coronavirus (2019-nCoV) Real-time RT-PCR Primers and Probes. National

602 Center for Immunization and Respiratory Diseases (NCIRD), Division of Viral Diseases, 2020.

603 (Accessed March 14, 2020, at https://www.cdc.gov/coronavirus/2019-ncov/lab/rt-pcr-

604 panel-primer-probes.html.)

605 32. Protocol: Real-time RT-PCR assays for the detection of SARS-CoV-2. Institut Pasteur,

606 2020. (Accessed July 6, 2020, at https://www.who.int/publications/m/item/molecular-

607 assays-to-diagnose-covid-19-summary-table-of-available-protocols.)

608 33. Corman VM, Landt O, Kaiser M, et al. Detection of 2019 novel coronavirus (2019-

609 nCoV) by real-time RT-PCR. Euro Surveill 2020;25:2000045.

610 34. Vogels CBF, Brito AF, Wyllie AL, et al. Analytical sensitivity and efficiency

611 comparisons of SARS-CoV-2 RT-qPCR primer-probe sets. Nat Microbiol 2020.

612 35. Arvia R, Sollai M, Pierucci F, Urso C, Massi D, Zakrzewska K. Droplet digital PCR

613 (ddPCR) vs quantitative real-time PCR (qPCR) approach for detection and quantification of

614 Merkel cell polyomavirus (MCPyV) DNA in formalin fixed paraffin embedded (FFPE)

615 cutaneous biopsies.

616 36. Penarrubia AL, Ruiz M, Porco R, et al. Multiple assays in a real-time RT-PCR SARS-

617 CoV-2 panel can mitigate the risk of loss of sensitivity by new genomic variants during the

618 COVID-19 outbreak. Int J Infect Dis 2020. 
619 37. Uhteg K, Jarrett J, Richards M, et al. Comparing the analytical performance of three

620 SARS-CoV-2 molecular diagnostic assays. J Clin Virol 2020;127:104384.

621 38. Etievant S, Bal A, Escuret V, et al. Performance Assessment of SARS-CoV-2 PCR

622 Assays Developed by WHO Referral Laboratories. J Clin Med 2020;9.

623 39. Kudo E, Israelow B, Vogels CBF, et al. Detection of SARS-CoV-2 RNA by multiplex RT-

624 qPCR. bioRxiv 2020:2020.06.16.155887.

625 40. Suo T, Liu X, Feng J, et al. ddPCR: a more accurate tool for SARS-CoV-2 detection in

626 low viral load specimens. Emerg Microbes Infect 2020;9:1259-68.

627 41. Xiao AT, Tong YX, Zhang S. Profile of RT-PCR for SARS-CoV-2: a preliminary study

628 from 56 COVID-19 patients. Clin Infect Dis 2020.

629 42. Zhou F, Yu T, Du R, et al. Clinical course and risk factors for mortality of adult

630 inpatients with COVID-19 in Wuhan, China: a retrospective cohort study. Lancet (London,

631 England) 2020;395:1054-62.

632 43. Zheng S, Fan J, Yu F, et al. Viral load dynamics and disease severity in patients

633 infected with SARS-CoV-2 in Zhejiang province, China, January-March 2020: retrospective

634 cohort study. Bmj 2020;369:m1443.

635 44. Lan L, Xu D, Ye G, et al. Positive RT-PCR Test Results in Patients Recovered From

636 COVID-19. JAMA 2020;323:1502-3.

637 45. Alvarez-Moreno CA, Rodríguez-Morales AJ. Testing Dilemmas: Post negative, positive

638 SARS-CoV-2 RT-PCR - is it a reinfection? Travel Med Infect Dis 2020;35:101743-.

639 46. Li N, Wang X, LV T. Prolonged SARS-CoV-2 RNA shedding: Not a rare phenomenon. J

640 Med Virol 2020.

641 47. Telwatte S, Lee S, Somsouk M, et al. Gut and blood differ in constitutive blocks to

642 HIV transcription, suggesting tissue-specific differences in the mechanisms that govern HIV

643 latency. PLoS Pathog 2018;14:e1007357.

644 48. Wölfel R, Corman VM, Guggemos W, et al. Virological assessment of hospitalized

645 patients with COVID-2019. Nature 2020;581:465-9.

646 49. Koev G, Miller WA. A positive-strand RNA virus with three very different subgenomic

647 RNA promoters. J Virol 2000;74:5988-96.

648 50. Pasternak AO, Spaan WJ, Snijder EJ. Nidovirus transcription: how to make sense...? J

649 Gen Virol 2006;87:1403-21.

650 51. Gorbalenya AE, Enjuanes L, Ziebuhr J, Snijder EJ. Nidovirales: evolving the largest

651 RNA virus genome. Virus Res 2006;117:17-37.

652 52. Baric RS, Yount B. Subgenomic negative-strand RNA function during mouse hepatitis 653 virus infection. J Virol 2000;74:4039-46.

654 53. Di H, Madden JC, Jr., Morantz EK, et al. Expanded subgenomic mRNA transcriptome

655 and coding capacity of a nidovirus. Proc Natl Acad Sci U S A 2017;114:E8895-e904.

656 54. Kuang J, Yan X, Genders AJ, Granata C, Bishop DJ. An overview of technical

657 considerations when using quantitative real-time PCR analysis of gene expression in human

658 exercise research. PLoS One 2018;13:e0196438-e.

659 55. Miranda JA, Steward GF. Variables influencing the efficiency and interpretation of

660 reverse transcription quantitative PCR (RT-qPCR): An empirical study using Bacteriophage

661 MS2. J Virol Methods 2017;241:1-10.

662 56. Levesque-Sergerie J-P, Duquette M, Thibault C, Delbecchi L, Bissonnette N. Detection

663 limits of several commercial reverse transcriptase enzymes: impact on the low- and high-

664 abundance transcript levels assessed by quantitative RT-PCR. BMC Mol Biol 2007;8:93-.

665 
bioRxiv preprint doi: https://doi.org/10.1101/2021.01.12.425991; this version posted January 12, 2021. The copyright holder for this preprint (which was not certified by peer review) is the author/funder, who has granted bioRxiv a license to display the preprint in perpetuity. It is made available under aCC-BY-NC-ND 4.0 International license.

666 Tables and Figures

667 Table 1. SARS-Cov2 ddPCR assay panel for assessing patient samples

668

\begin{tabular}{lll}
\hline \multicolumn{1}{c}{ Assay Name } & \multicolumn{1}{c}{ RNA Target } & \multicolumn{1}{c}{ Detects } \\
\hline 5'UTR & 5' untranslated region & Genomic RNA \\
Main Proteinase-NSP5 & Main Proteinase & Genomic RNA \\
RdRp & RNA-dependent RNA Polymerase & Genomic RNA \\
S-PBCS & $\underline{\text { Polybasic cleavage site of the surface }}$ & Genomic/subgenomic \\
M-ORF5 & (S) glycoprotein & \\
N-ORF9 & Membrane glycoprotein & Genomic/subgenomic \\
3'UTR & Nucleocapsid & Genomic/subgenomic \\
& 3' untranslated region & Genomic/subgenomic
\end{tabular}

669

670 
671 Table 2. SARS-CoV-2 primer/probe sets selected for validation using IVT and virion

672 RNA

\begin{tabular}{llll}
\hline $\begin{array}{l}\text { Target } \\
\text { Region }\end{array}$ & Primer Name $^{\text {a }}$ & $\begin{array}{l}\text { SARS-CoV-2 } \\
\text { coordinates }^{\mathbf{b}}\end{array}$ & \multicolumn{1}{c}{ Sequence (5'-3') } \\
\hline 5'UTR & & & \\
& 5'UTR_F & $152-171$ & GTTGACAGGACACGAGTAAC \\
& 5'UTR_P & $175-197$ & TCTATCTTCTGCAGGCTGCTTAC \\
& 5'UTR_R & $220-241$ & GAAACCTAGATGTGCTGATGAT \\
\hline
\end{tabular}

Main proteinase/NSP5 (ORF1a)

\begin{tabular}{lll} 
NSP5_F & $10366-10387$ & TCGCATTCAACCAGGACAGACT \\
NSP5_P & $10399-10425$ & AGCTTGTTACAATGGTTCACCATCTGG \\
NSP5_R & $10426-10450$ & GGGCCTCATAGCACATTGGTAAACA \\
\hline
\end{tabular}

RNA-dependent RNA polymerase / NSP12 (ORF1b)

RDRP_F 15341-15364 CCTCACTTGTTCTTGCTCGCAAAC

RDRP_P 15370-15393 ACGTGTTGTAGCTTGTCACACCGT

RDRP_R 15437-15456 TGAACCGCCACACATGACCA

$\mathrm{S}$ protein/ polybasic cleavage site (ORF 2)

S_PBCS_F 23554-23576 ACCCATTGGTGCAGGTATATGCG

S_PBCS_P 23603-23622 ACACTACGTGCCCGCCGAGG*

S_PBCS_R 23641-23664 GCACCAAGTGACATAGTGTAGGCA

$M$ protein (ORF 5)

$\begin{array}{lll}\text { M-ORF5_F } & \text { 26768-26789 } & \text { CGCAATGGCTTGTCTTGTAGGC } \\ \text { M-ORF5_P } & 26794-26816 & \text { TGTGGCTCAGCTACTTCATTGCT } \\ \text { M-ORF5_R } & 26821-26840 & \text { CGTACGCGCAAACAGTCTGA }\end{array}$

$\mathrm{N}$ protein (ORF 9)

$\begin{array}{lll}\text { N-ORF9_F } & 28833-28851 & \text { CATCACGTAGTCGCAACAG } \\ \text { N-ORF9_P } & 28885-28907 & \text { AACTTCTCCTGCTAGAATGGCTG } \\ \text { N-ORF9_R } & 28917-28934 & \text { AAGCAAGAGCAGCATCAC }\end{array}$

3'UTR

$\begin{array}{lll}\text { 3'UTR_F } & 29702-29723 & \text { GGAGGACTTGAAAGAGCCACCA } \\ \text { 3'UTR_P } & 29727-29746 & \text { TTTCACCGAKGCCACRCGGA } \\ \text { 3'UTR_R } & 29768-29788 & \text { GGCAGCTCTCCCTAGCATTGT }\end{array}$

$673{ }^{*}$ Reverse complement

674 a 'F' = forward primer, 'R' = reverse primer, 'P'= probe (fluorophore/quencher: FAM, MGB)

675 bSARS-CoV2 coordinates indicated are based on the SARS-CoV2 reference sequence

676 (NC_045512.2)

677 
678 Table 3. SARS-CoV-2 assays from other sources

\begin{tabular}{|c|c|c|c|c|}
\hline $\begin{array}{l}\text { Target } \\
\text { Region }\end{array}$ & Primer Name $^{a}$ & $\begin{array}{l}\text { SARS-CoV-2 } \\
\text { coordinates }^{\text {b }}\end{array}$ & Sequence (5-3') & Reference \\
\hline \multirow{7}{*}{$\begin{array}{l}\mathrm{N} \\
\text { protein/ } \\
\text { ORF } 9\end{array}$} & & & & \multirow{7}{*}{31} \\
\hline & CDC_N1_F & $28287-28306$ & GAC CCC AAA ATC AGC $\underline{\text { GAA AT }}$ & \\
\hline & CDC_N1_P & $28309-28330$ & 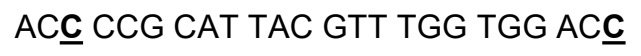 & \\
\hline & CDC_N1_R & $28335-28358$ & TCT GGT TAC TGC CAG TTG AAT CTG & \\
\hline & CDC_N2_F & $29164-29183$ & TTACAAACATTGGCCGCAAA & \\
\hline & CDC_N2_P & 29188- 29210 & ACAATTTGCCCCCAGCGCTTCAG & \\
\hline & CDC_N2_R & $29213-29230$ & GCG CGA CAT TCC GAA GAA & \\
\hline \multirow[t]{3}{*}{ ORF1a } & $\begin{array}{l}\text { nCoV_IP2- } \\
12669 \mathrm{Fw} \\
\text { nCoV IP2- }\end{array}$ & $12690-12707$ & ATGAGCTTAGTCCTGTTG & \multirow[t]{3}{*}{32} \\
\hline & $\begin{array}{l}\text { 12696̄bProbe }(+) \\
\text { nCoV_IP2- }\end{array}$ & $12717-12737$ & AGATGTCTTGTGCTGCCGGTA & \\
\hline & $12759 \mathrm{Rv}$ & $12780-12797$ & CTCCCTTTGTTGTGTTGT & \\
\hline \multirow[t]{3}{*}{ E gene } & E_Sarbeco_F1 & 26269-26394 & ACAGGTACGTTAATAGTTAATAGCGT & \multirow{3}{*}{33} \\
\hline & E_Sarbeco_P1 & $26332-26357$ & ACACTAGCCAICCTTACTGCGCTICG & \\
\hline & E_Sarbeco_R2 & $26360-26381$ & ATATTGCAGCAGTACGCACACA & \\
\hline
\end{tabular}

679 a 'F' = forward primer, 'R' = reverse primer, 'P'= probe (fluorophore/quencher: FAM, MGB)

680 b SARS-CoV-2 coordinates indicated are based on the SARS-CoV-2 reference sequence

681 (NC_045512.2)

682 Bold and underlined $=$ known mismatches as reported in ${ }^{34}$ and in-house SARS-CoV2

683 multiple sequence alignment (mismatches identified were relative to sequence MT825091.1

684 from Iran) 
686 Figure 1. Schematic presentation of SARS-CoV2 genome organization, virion

687 structure and canonical sgRNAs. SARS-CoV-2 encodes two large genes, ORF1a (yellow)

688 and ORF1b (blue), which encode 16 non-structural proteins (NSP1-NSP16). The structural

689 genes encode the structural proteins, spike (S; green), envelope (E; blue), membrane (M;

690 purple), and nucleocapsid ( $\mathrm{N}$; gold). Assay locations of each assay designed for this study

691 are indicated. Virion structure and canonical subgenomic RNAs produced by SARS-CoV-2

692 are shown in the lower panel (S, 3a, E, M, 6, 7a, 7b, 8 and N).

693

694

Figure 2. Efficiency and linearity of SARS-CoV-2 panel of ddPCR assays determined

using plasmid DNA. Plasmids containing individual SARS-CoV-2 genes or regions were

quantified by UV spectroscopy and diluted (expected copies) to test the absolute number of

697 copies detected by each primer/probe set using duplicate ddPCR reactions (measured copies). Two primer/probe sets were tested for each region except the S-PBCS. One primer/probe set from each region (indicated by coloured symbol) was selected for subsequent experiments.

701

702 Figure 3. Efficiency and linearity of SARS-CoV-2 panel of ddPCR assays determined using in vitro transcribed (IVT) RNA. RNA standards containing a given region or gene of SARS-CoV-2 were prepared by in vitro transcription from plasmids and quantified by independent means (UV spectroscopy and the Agilent Bioanalyzer). Various inputs of each IVT RNA standard (which were used to calculate 'Expected Copies' per ddPCR well) were reverse transcribed and replicate aliquots of cDNA were used to measure the absolute number of copies detected by each ddPCR assay ('Measured Copies'). Each assay was tested using expected inputs of $1-10^{4}$ copies per ddPCR well (except S-PBCS, which was tested at inputs independent experiments. 
714 Figure 4. Efficiency and linearity of SARS-CoV2 panel of ddPCR assays determined

715 using SARS-CoV-2 virion RNA. A SARS-CoV-2 "virion" standard was prepared by

716 extracting the RNA from the supernatant of an in vitro infection and quantified using the Abbott

717 Real Time SARS-CoV-2 assay. Various inputs of the virion standard (which were used to

718 calculate 'Expected Copies’ per ddPCR well) were applied to a common reverse transcription

719 reaction, from which aliquots of cDNA were used to measure the absolute number of copies

720 detected by each ddPCR assay (measured copies). Each assay was tested with expected

721 inputs of 10-10 copies/ddPCR well in duplicate. S (slope) and $\mathrm{R}^{2}$ are indicated for each assay.

722 Representative data for $n=2$ independent experiments are shown.

723

724 Figure 5. Comparison of assay efficiency and linearity of published assays, ORF1a

725 “nCoV_IP2" and E gene and novel RDRP-NSP12 assay. The performance of our RDRP-

726 NSP12 assay was compared to published primers/probes for ORF1a and the E gene in the

727 ddPCR platform using common RT reactions containing virion standard RNA inputs of 2-2x10

728 copies/ddPCR well. S (slope) and $\mathrm{R}^{2}$ are indicated for each assay.

729

730 Figure 6. Effect of background RNA on ddPCR assay performance. We simultaneously

731 tested all assays in our panel against reported assays, CDC_N1, CDC_N2, E_Sarbeco, and

732 IP2_ORF1a, in the presence and absence of background RNA. Each assay was tested with

733 a constant input of SARS-CoV-2 virion standard (predicted to yield 1000 copies/ddPCR well)

734 in the presence or absence of background RNA from PBMC or a lung epithelial cell line (A549)

735 added at a concentration of $100 \mathrm{ng} / \mu \mathrm{L}$ of $\mathrm{RT}$ reaction $(500 \mathrm{ng} / \mathrm{ddPCR}$ well, or $1 \mu \mathrm{g}$ for the 2

736 replicate wells used to test each assay). Negative controls included water, $1 \mu \mathrm{g} /$ assay PBMC

737 RNA, and 1 mg/assay A549 RNA. Assays are indicated on x-axis in order from 5' to 3' and

738 dotted line indicates 1000 SARS-CoV-2 RNA copy input. Error bars represent standard

739 deviation from duplicate wells. 
741 Figure 7. Transcription profile of three SARS-CoV-2 infected individuals determined

742 using RT-ddPCR. Unused nucleic acid (ranging from 8.6-16.8 $\mu \mathrm{L}$ ) extracted by the Abbott

743 m2000 platform from nasopharyngeal swabs from SARS-CoV-2 infected individuals $(n=3)$

744 was used in a common RT reaction for each individual. Resulting cDNA was divided evenly

745 across reactions for the seven assays in our panel and targets were measured using

746 ddPCR. Colored symbols indicate SARS-CoV-2 target region. Copy numbers from each

747 assay are expressed as SARS-CoV-2 copies per $\mu \mathrm{L}$ of nucleic acid and grouped for each

748 individual ( $\mathrm{x}$-axis). Threshold cycle $\left(\mathrm{C}_{\mathrm{t}}\right)$ values, as determined by Abbott Real Time SARS-

749 CoV-2 viral load assay, are indicated above each individual's dataset. 


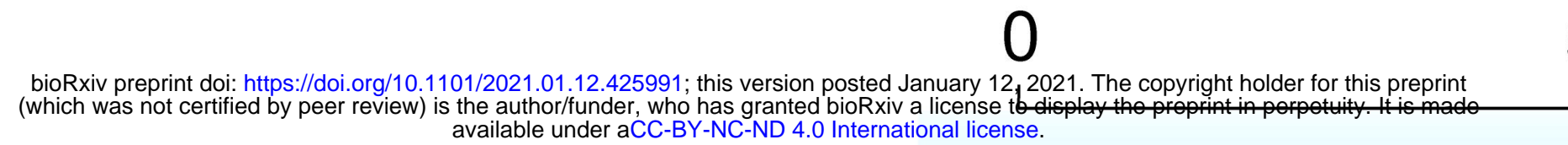

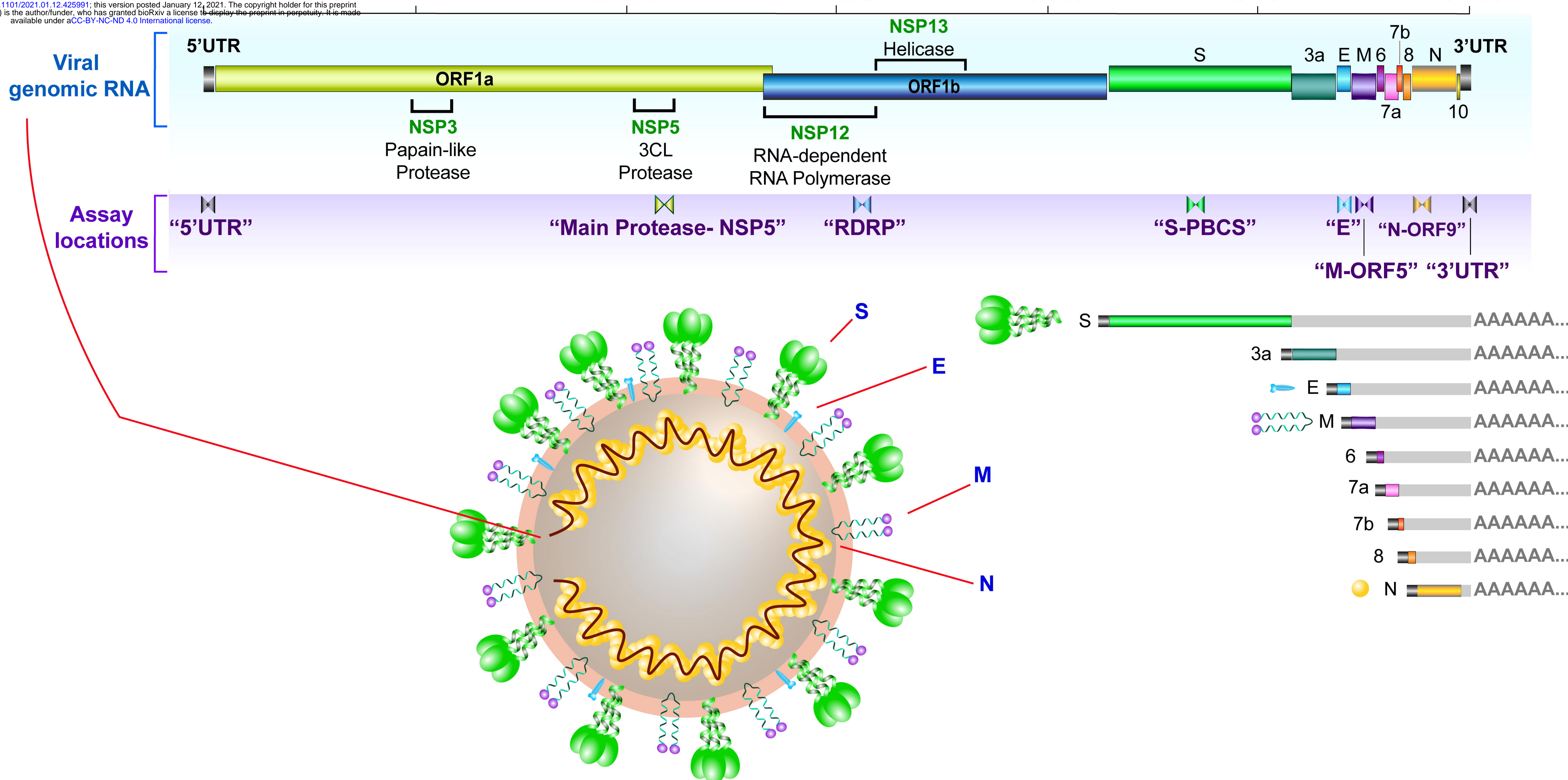




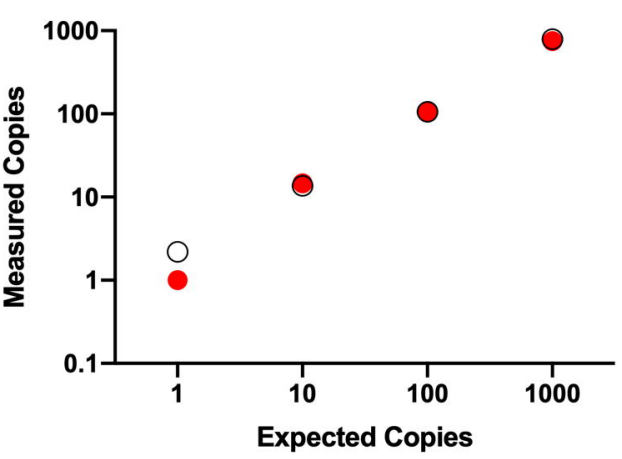

RNA-dependent RNA Polymerase

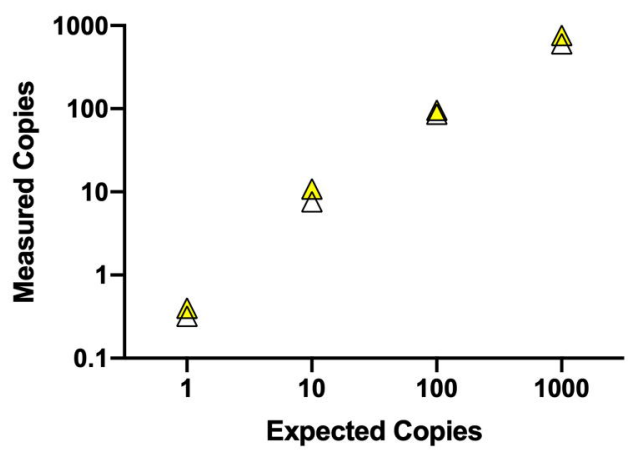

M-ORF5

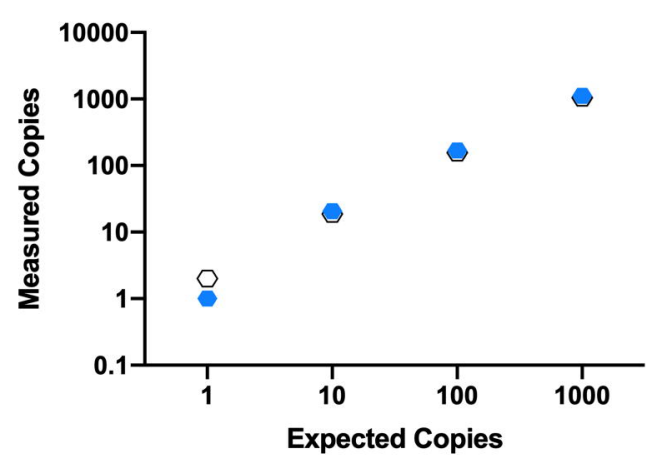

3'UTR

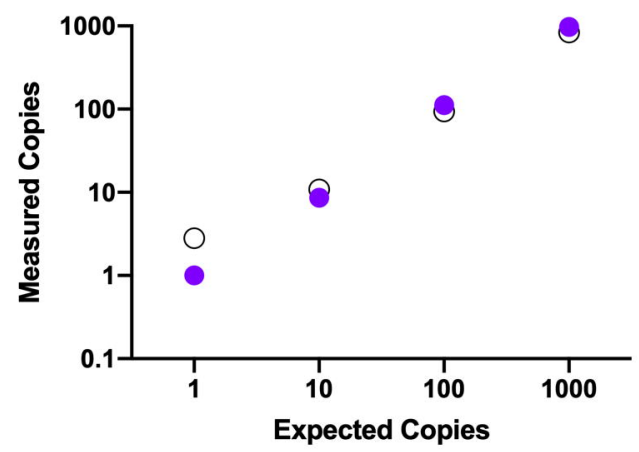

5'UTR_A

$R^{2}=0.9986$

Slope $=0.74$

O 5'UTR_B

$R^{2}=0.9990$

Slope $=0.78$

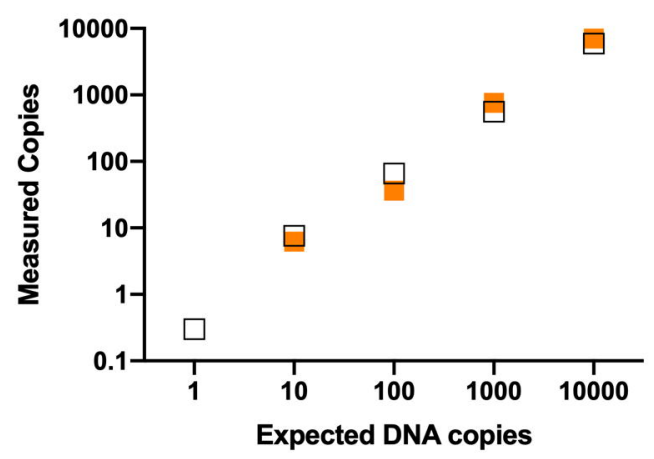

S-PBCS

$\triangle$ RDRP-NSP12_A $\mathrm{R}^{2}=0.9994$ Slope $=0.76$

$\triangle$ RDRP-NSP12_B $\mathrm{R}^{2}=0.9983$ Slope $=0.60$

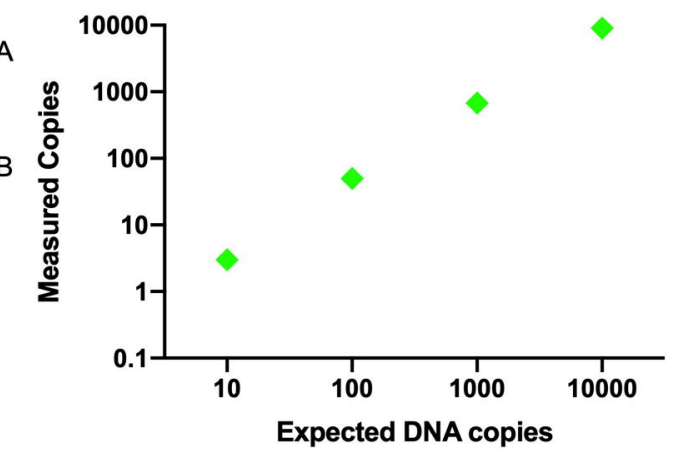

N-ORF9

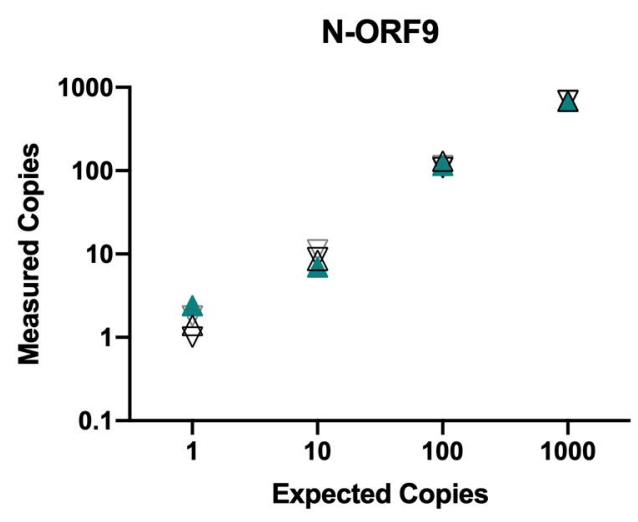

- M_ORF5_A Slope=1.1

○ M_ORF5_B $R^{2}=0.9978$ Slope $=1.1$ $\mathrm{R}^{2}=0.9 \overline{9} 8$
Pro-Nsp5_A $R^{2}=0.9999$ Slope $=0.70$

Pro-Nsp5 B $\mathrm{R}^{2}=1.0$ Slope $=0.59$
S-PBCS_A $R^{2}=0.9995$ Slope $=0.91$

- N-ORF9 A $\mathrm{R}^{2}=0.9951$ Slope $=0.67$

$\triangle$ N-ORF9_B $\mathrm{R}^{2}=0.9 \overline{9} 18$ Slope $=0.67$

$\nabla$ CDC_N1 $\mathrm{R}^{2}=0.9940$ Slope $=0.68$ $\checkmark$ CDC_N2 $\mathrm{R}^{2}=\overline{0} .9957$ Slope $=0.67$

- 3'UTR_A $R^{2}=0.9997$ Slope $=0.97$

O 3'UTR_B $R^{2}=0.9999$ Slope $=0.82$ 


\section{Panel of SARS-CoV2 ddPCR assays}

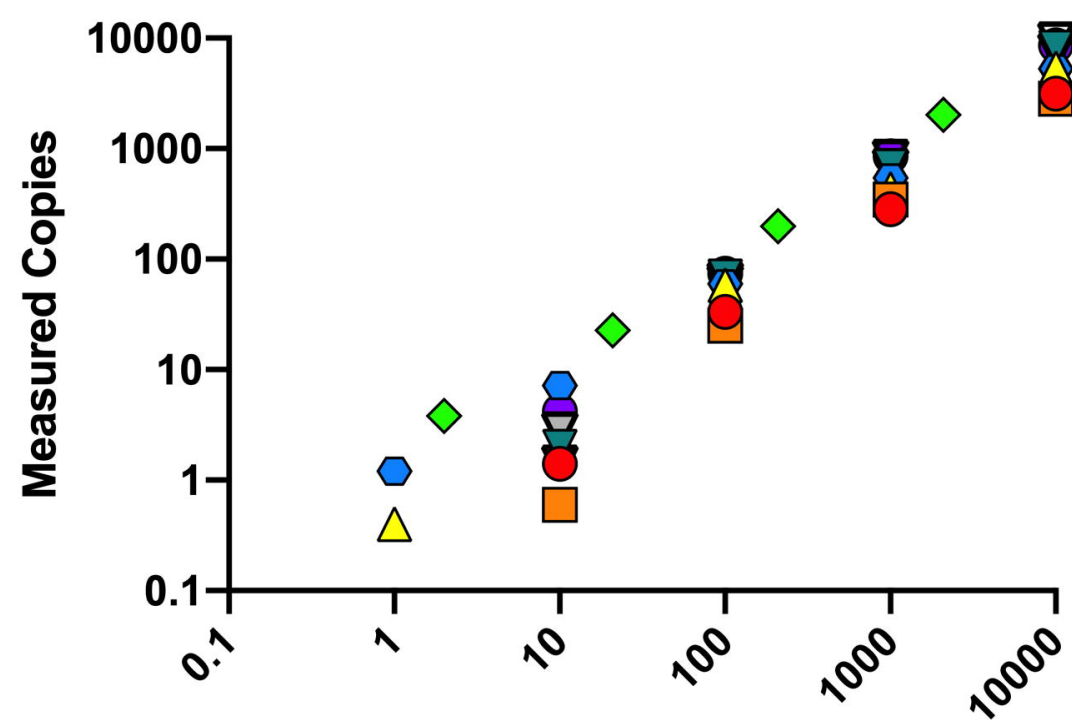

○'UTR

$S=0.36$

$\square$ Main Proteinase-NSP5 $S=0.18$

$\triangle$ RdRp-Nsp12

$S=0.53$

$\diamond$ S-PBCS

$S=0.96$

- M-ORF5

$S=0.53$

$\nabla \quad \mathrm{N}-\mathrm{ORF9}$

$S=0.82$

$\nabla$ CDC_N1

$S=0.96$

$\nabla$ CDC_N2

$S=0.75$

○'UTR

$S=0.86$

\section{Expected Copies}



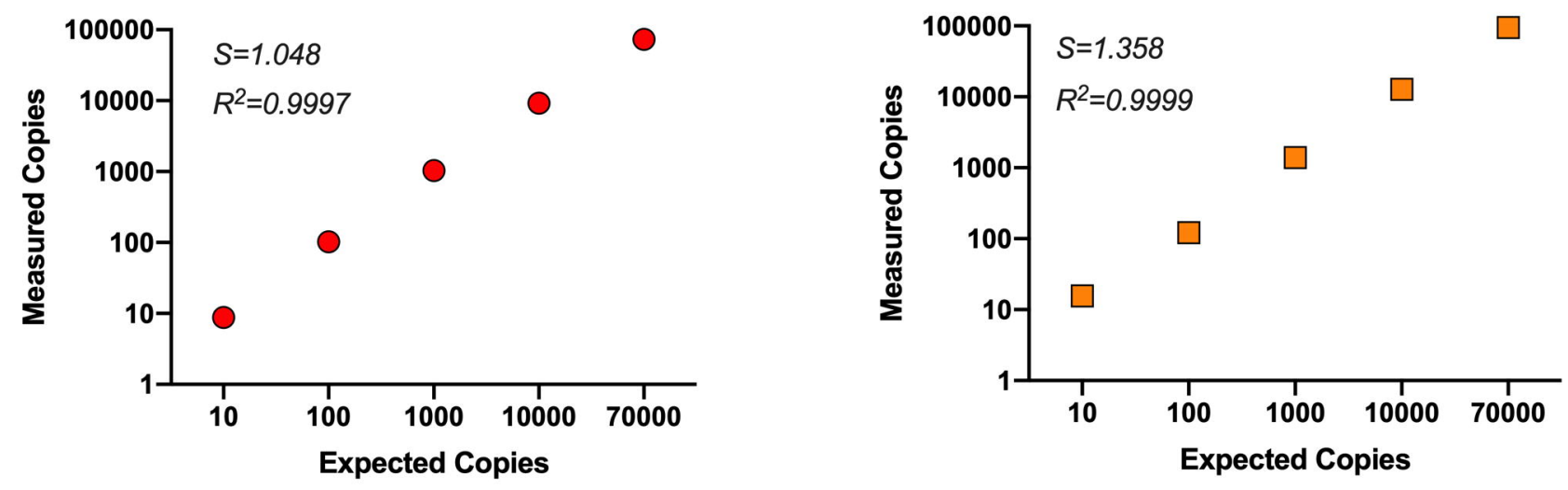

RdRP

S-PBCS
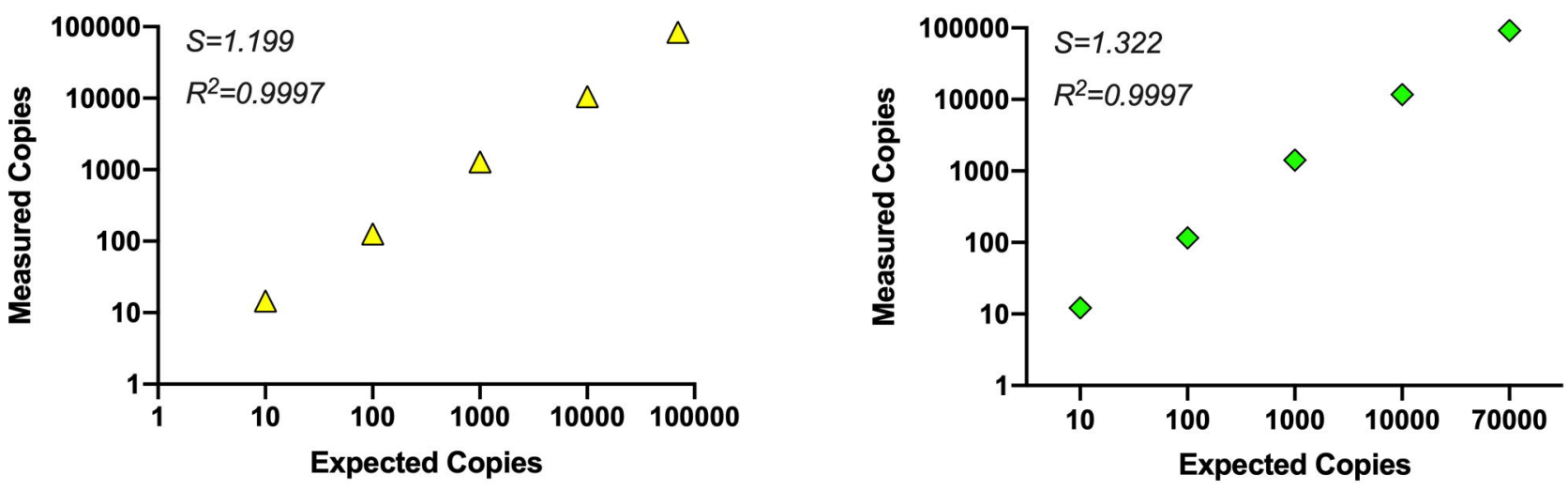

\section{M-ORF5}

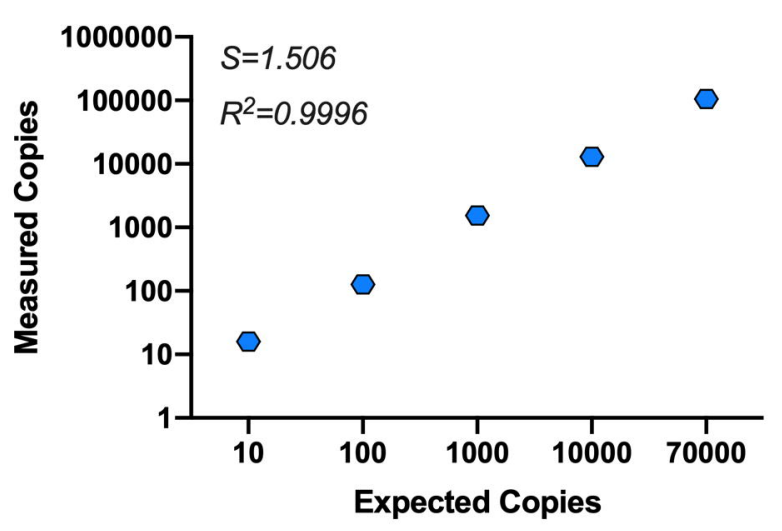

3'UTR

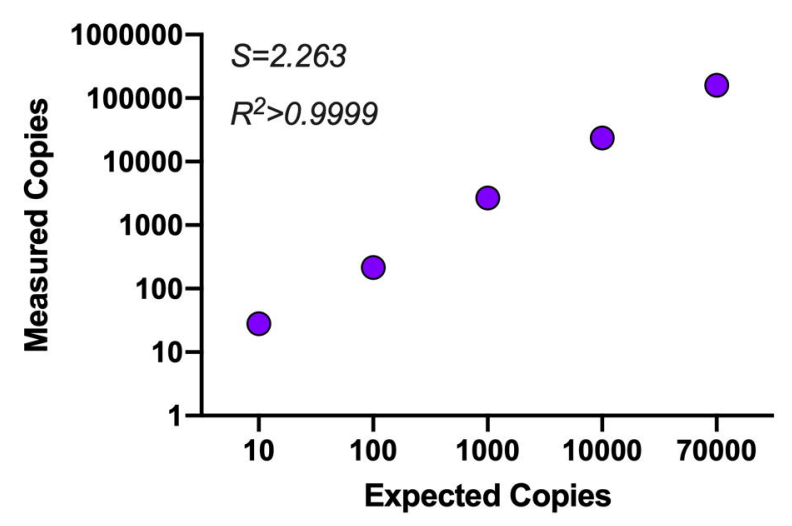


IP2_ORF1a

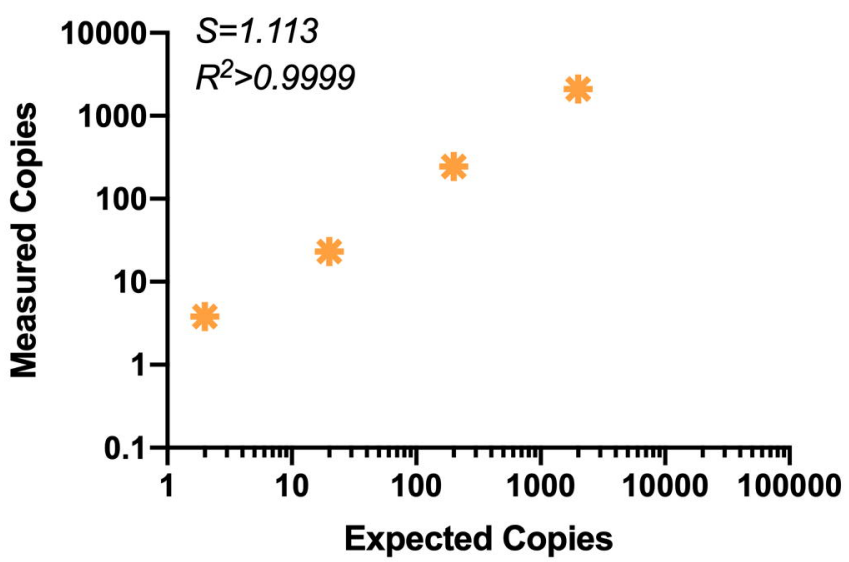

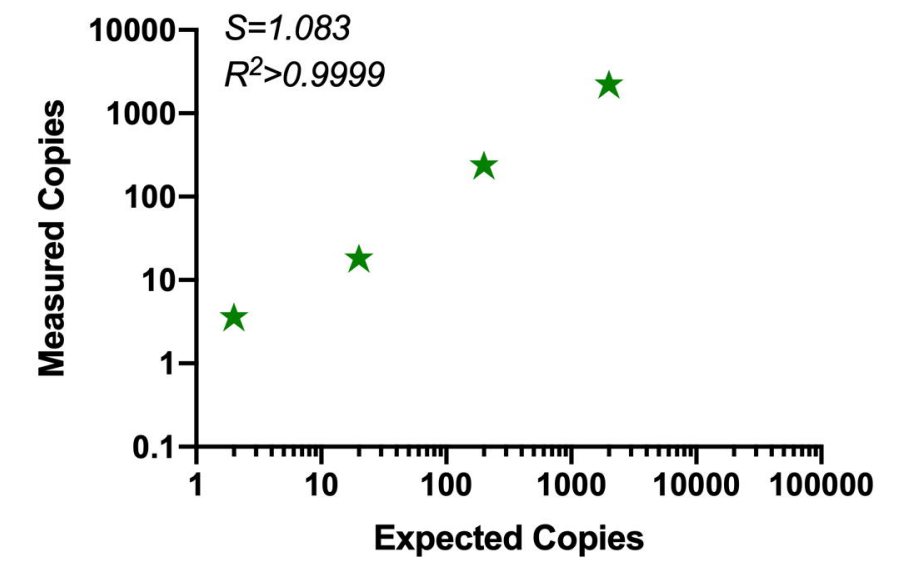




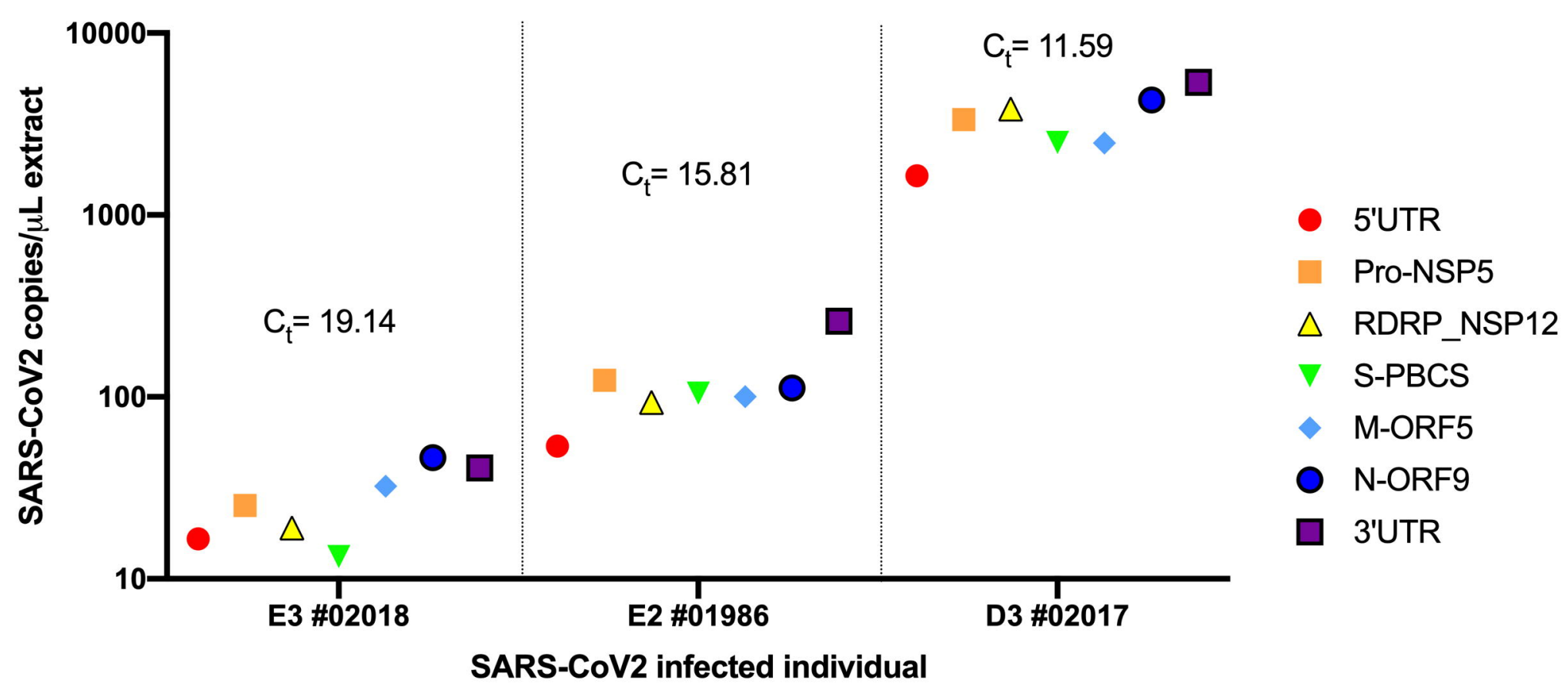

